\title{
Respiratory Rate Derived from Principal Component Analysis of Single Lead Electrocardiogram
}

\author{
EJ Bowers $^{1}$, A Murray ${ }^{1,2}$, P Langley $^{1,2}$ \\ ${ }^{1}$ Freeman Hospital, Newcastle upon Tyne, UK \\ ${ }^{2}$ Newcastle University, Newcastle upon Tyne, UK
}

\begin{abstract}
We used principal component analysis to derive the respiratory rate from single lead ECGs. In this algorithm the respiratory induced beat-to-beat variability of the $E C G$ is described by the coefficients of the principal components.

Subjects were asked to breathe at different rates and naturally while respiration and ECG were recorded. Breathing rate, determined by Fourier analysis, was compared for the ECG derived respiration obtained by principal component analysis and the recorded respiratory signal.

Across the different breathing patterns the mean absolute differences between reference respiratory rate and ECG respiratory rate were 0.2 breaths per minute (bpm) or less. In all cases the respiratory rate was accurately determined from respiratory surrogates derived from principal component analysis of the single lead ECGs.
\end{abstract}

\section{Introduction}

There are a number of applications which require the simultaneous monitoring of respiration and ECG, including the clinical investigation of sleep disorders and sports performance monitoring. Minimising the number of sensors required to perform these measurements is an important consideration. Respiratory induced changes in the ECG make it possible to derive a surrogate respiratory signal directly from the ECG, potentially eliminating the need for a separate respiratory sensing system. A number of algorithms for ECG derived respiration have already been presented in the literature [1]. Here we demonstrate the application of principal component analysis to derivation of a surrogate respiratory signal from single lead ECGs from which respiratory rate can be estimated.

\section{Methods}

Single lead ECG and respiration were recorded during controlled and natural breathing in 10 healthy subjects.
Respiration was monitored using magnetic displacement sensors placed on the back and chest of the subjects. Lead II of the ECG was recorded. During these recordings subjects were asked to breathe at different rates up to $14 \mathrm{bpm}$ and at their natural breathing rate for periods of 3 minutes. For controlled breathing, subjects were asked to synchronise their breathing to a visual respiratory control signal which oscillated at the required breathing rate. Both ECG and respiration were digitally recorded at a sample rate of $500 \mathrm{~Hz}$.

To derive the respiratory signal from the ECG using the principal component algorithm the following steps were undertaken.

i) Beat detection: $\mathrm{R}$ wave peaks at each beat were identified using a beat detection algorithm.

ii) Beat collection: the beats were collected time ordered into a matrix $(x)$ in which all beats were time aligned to the $\mathrm{R}$ wave peaks. $x$ is a $m \times n$ matrix with $m$ corresponding to the number of beats and $n$ corresponding to the number of samples per beat. The samples were determined by a fixed window of $n$ sample points around the $\mathrm{R}$ wave peak such that the window included $\mathrm{P}$ wave, QRS complex and T wave.

iii) Principal component analysis: principal component analysis was applied to matrix $x$ to yield $m$ principal components and for each principal component a series of $m$ coefficients. These coefficients effectively describe the beatto-beat variability of the principal components and provide the ECG derived respiration in this algorithm.

Figure 1 illustrates the process. Fourier analysis was applied to the measured respiratory signal and to the ECG derived respiratory signal. Each heart beat provides an irregular sample point for the ECG derived respiratory signal so it was necessary to resample this to a regular sample rate using linear interpolation before applying 
spectral analysis. Respiration rate was identified from the frequency of the spectral peak with the largest power. respiratory rates were compared. The resolution of the spectral analysis was $0.342 \mathrm{bpm}$.

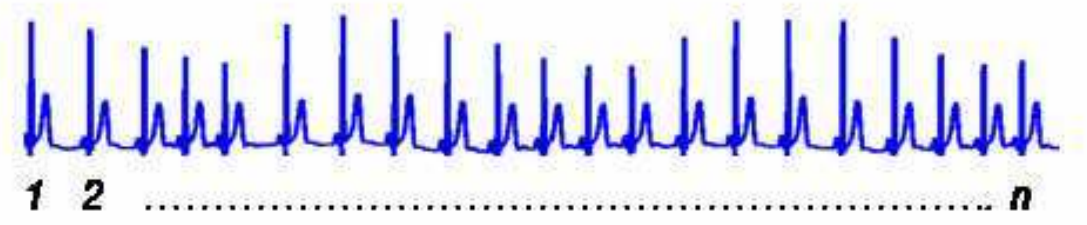

Beat No.

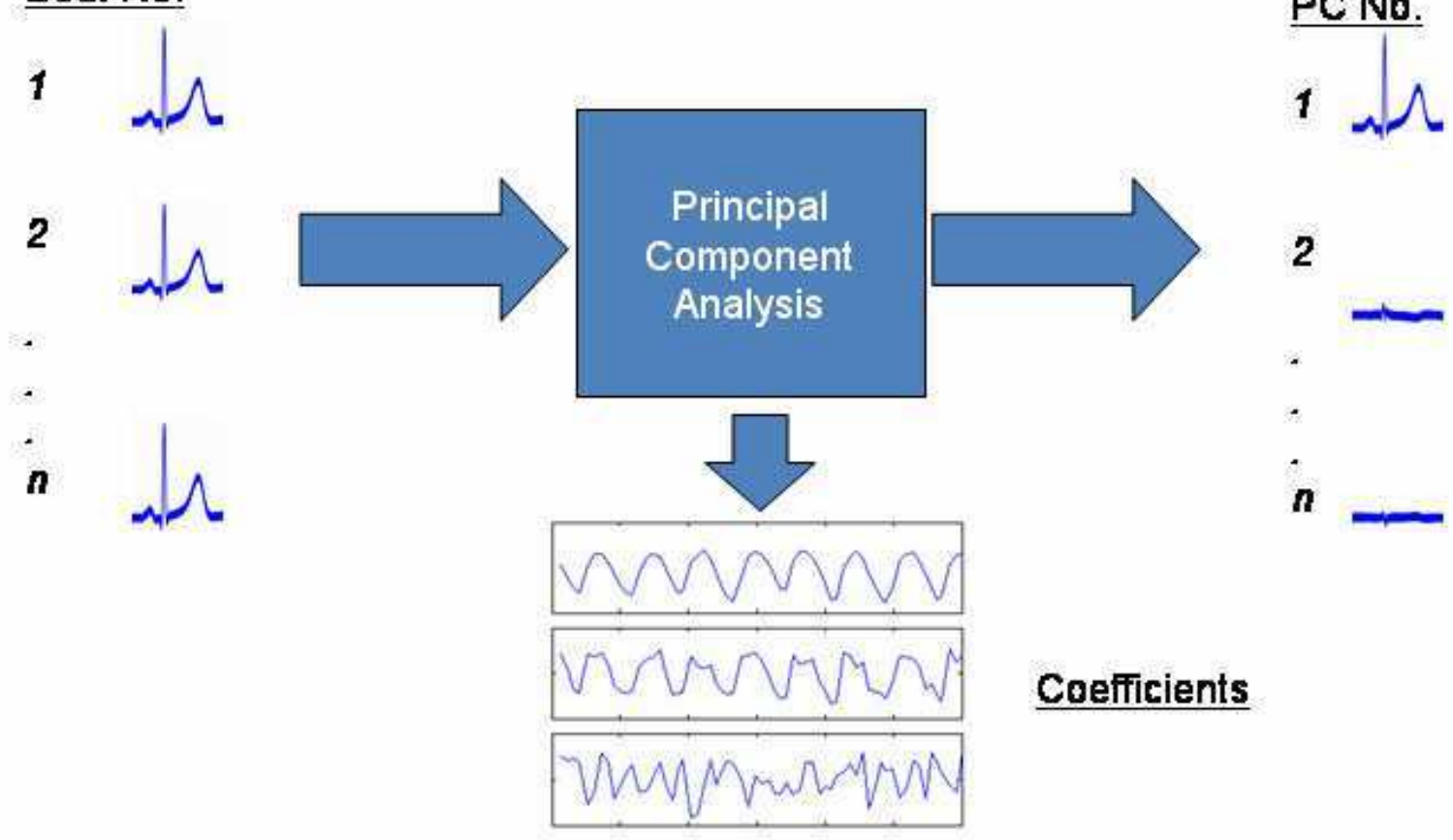

Figure 1. Illustration of the application of principal component analysis to deriving respiration from the single lead ECG. Top: single lead ECG. Left: Collection of ECG beats. Middle: Principal component analysis is applied to the time-aligned collection of beats. Right: Principal components (PC). Bottom: The first three principal component coefficients. The coefficients of the first principal component provide the respiratory surrogate.

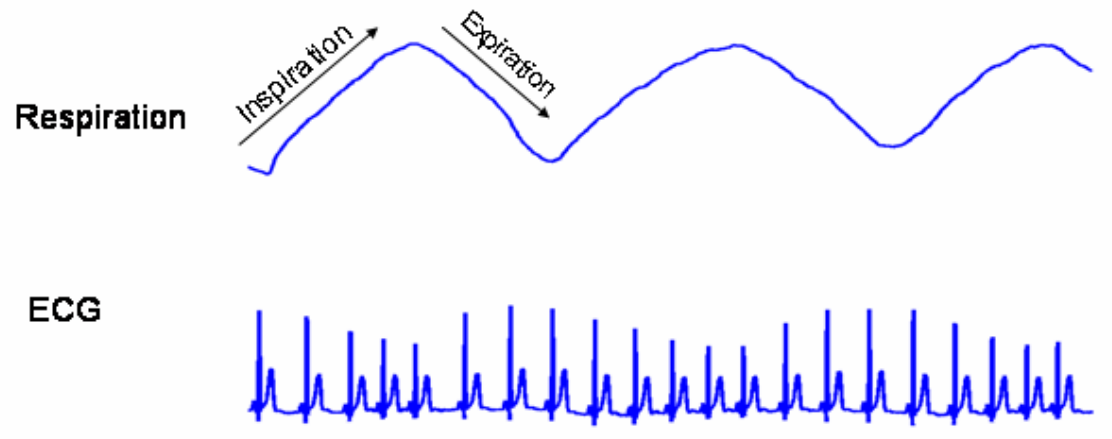

Figure 2. Illustration of respiratory effects on the ECG. The respiratory signal measured as chest displacement is shown above the simultaneously recorded the ECG. The amplitude and inter-beat interval of the ECG are modulated at a rate corresponding to the respiratory rate. Duration of the signals is $20 \mathrm{~s}$. 

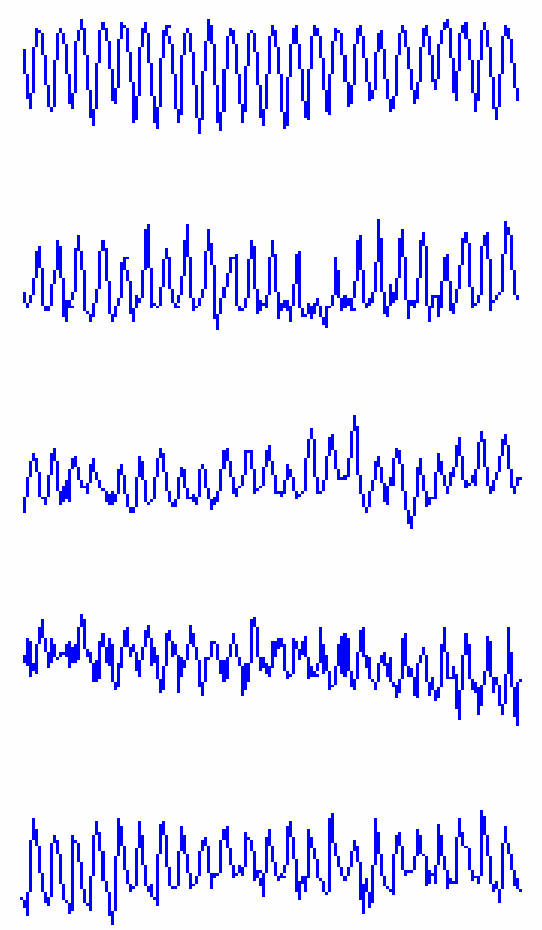
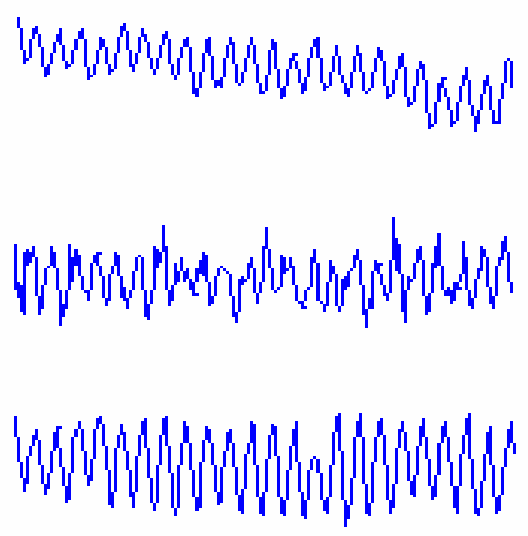

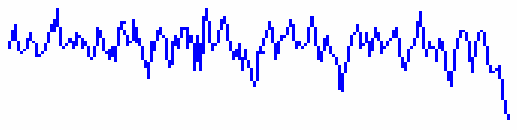

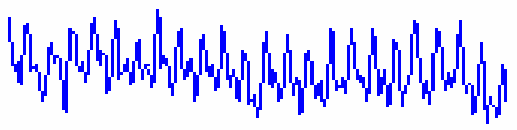

Figure 3. ECG derived respiration from all subjects at a breathing rate of $8 \mathrm{bpm}$. Duration is $180 \mathrm{~s}$ and amplitudes are normalized to peak to peak amplitudes.

\section{Results}

Figure 2 shows an example of the respiration signal obtained from the magnetic displacement sensors and the simultaneously recorded ECG from a subject breathing at $8 \mathrm{bpm}$. The amplitude of the $\mathrm{R}$ wave and the RR interval are clearly modulated at a rate corresponding to the respiratory signal.

Spectral analysis of the respiratory signals showed that subjects followed the requested controlled breathing rates closely with differences of $0.1 \mathrm{bpm}$ or less.

Mean absolute differences between reference respiratory rate and ECG respiratory rate were $0.2 \mathrm{bpm}$ or less. The natural breathing rate ranged from 7 to $15 \mathrm{bpm}$ between subjects and the mean absolute difference between reference and ECG respiratory rate was also less than $0.2 \mathrm{bpm}$. Figure 3 illustrates the coefficients of the first principal component of the ECG for all subjects breathing at a rate of $8 \mathrm{bpm}$.

The respiratory rates obtained from the principal component derived respiration were accurate and the differences between these and the measured respiratory signals were less than spectral resolution of the rate measurements.

\section{Discussion and conclusions}

The principal component coefficients obtained from the application of principal component analysis to beats from the single lead ECG provide a surrogate for the respiratory signal from which respiratory rate can be accurately estimated. The coefficients describe the beatto-beat variability of ECG morphology. Here we used the coefficients of the first principal component to derive the respiratory signal but there were also respiratory related changes in the coefficients of some of the lower principal components. This is illustrated in figure 1 where the coefficients of not only the first but also the second principal component clearly show respiratory related changes. The algorithm was applied to the full ECG cycle comprising $\mathrm{P}$ wave, QRS complex and T wave, however, the algorithm could also be applied to these features individually to investigate the beat-to-beat variability of these features. As an example, we applied 
this algorithm in the 2008 Computers in Cardiology Challenge for $\mathrm{T}$ wave alternans detection [2]. Further work will be required to assess the algorithm for use in more demanding applications such as exercise testing and to test the algorithm on different leads.

\section{Acknowledgements}

Philip Langley is supported by an EPSRC Advanced Research Fellowship.

\section{References}

[1] Bailón R, Sörnmo L, Laguna P. ECG-derived respiratory frequency estimation. In: Clifford GD, Azuaje F,
McSharry PE, editors. Advanced methods and tools for ECG data analysis. London: Artech House, 2006;215-43.

[2] Zheng D, Stevens S, Langley P, Wang K, Haigh AJ, Murray A. T-wave alternans: A comparison of different measurement techniques. Comput Cardiol 2008;35(in press).

Address for correspondence

Philip Langley

Medical Physics Dept, Freeman Hospital, Newcastle upon Tyne, UK

philip.langley@ncl.ac.uk 